\title{
Use of Kinematic Analysis For Rational Choice of Parameters and Modes of Tillage Disc
}

\author{
A. P. Akimov, V. I. Medvedev, V. P. Mazyarov \\ Professor, Department of Engineering \\ Chuvash state agricultural Academy \\ Cheboksary, Russia \\ akimov_mechfak@mail.ru
}

\author{
G. S. Yunusov \\ Department of Mechanization of Production and Processing \\ of Agricultural Products \\ Mari State University \\ Yoshkar-Ola, Russia \\ kafmeh@yandex.ru
}

\author{
Yu. V. Konstantinov \\ Associate Professor, Department of Engineering \\ Chuvash state agricultural Academy \\ Cheboksary, Russia \\ konstantinov@polytech21.ru
}

\author{
L. M. Rybakov \\ Department of power supply and technical diagnostics \\ Mari State University \\ Yoshkar-Ola, Russia \\ naukamarsu@bk.ru
}

\begin{abstract}
The paper gives a method to determine a blade line element cutting angle of tillage inclined and vertical flat disks. Formulas are obtained for the blade line element cutting angle of discs, depending on its geometric and operating parameters. Similar dependencies based on the angle, characterizing soil ripping capability of any elementary disc surface area, were determined. Based on these dependencies, it was demonstrated that the vertical discs crumble the soil better than inclined ones. For small depth for the angle, characterizing ripping capability, they are closer to plows and go far beyond rotatory cultivators. Using the dependencies for blade line element cutting angles of discs, the authors demonstrated the way one can narrow the scope for rational choice of geometrical and mode parameters of disks and thus reduce the scope and duration of necessary experimental studies. For an optimal range of all blade line element cutting angles, interacting with the soil, one need either brake disks hard enough or force them to rotate.
\end{abstract}

Keywords - disk, cultivation, cutting angle, tillage

\section{INTRODUCTION}

Disk till-plows are used not only for grain and other crops primary tillage, but also for secondary and out of crop tillage. Plows with flat discs are preferable for the last two types of work as they spray soil less and do not bring its bottom (wet) layers to the surface. In addition, when processing stubble fields with flat discs, a large part of the crop residues on the surface allows keeping moisture, so the till-plows with such discs are commonly used in areas prone to soil wind erosion. Since 1968, 30\% of till-plows of the LD-10 were manufactured with flat disks [1].

For more efficient use of disks, slow or active rotation modes are used in the tillage. Till-plow discs slowing down enables to improve their agrotechnical and energy performance when operating at higher speeds [2]. Forced discs rotation from the tractor PTO shaft is used in the harrow of
"Brown" company (UK), which is used for stubble primary and secondary tillage [3].

It was also suggested to set discs not vertically but at some acute angle to the daylight field surface to increase operating speeds and improve qualitative operation characteristics of the till-plow. Such discs swing and turn the soil much less, but better [4].

It is necessary to know dependence of their power characteristics from the geometric and operating parameters of the discs to design such tools with the cultivation disks. However, works on their experimental determination are relatively rare $[1,3]$, hence a theoretical analysis of the interaction of tillage disc with the soil is of great importance. It reduces the amount of necessary experiments based on their proper planning.

Optimal parameters and operation modes of the rotary work tools of tillage machines and tools ensure quality tillage with minimum specific energy consumption $[5,6]$. It is necessary to analyze interaction of work tools with the soil to determine such optimal combination. This task is quite difficult, and designers often have to confine themselves to their kinematic analysis, studying the movement of rotary work tools less regard to applied forces. Even a relatively simple analysis in some cases allows reasonable choosing of constructive and technological parameters of the work tools by narrowing the range of allowable values for these parameters.

The first step of kinematic analysis is the determination of laws of motion of the rotary work tools, including disk, their elements and points. A number of authors are limited to such laws of motion [7]. Set laws of rotary work tools points' motion allow setting a motion path of these points. And the analysis of these paths allows to make important conclusions on the quality of the soil, in particular, to determine the dependence of the plow sole irregularities from the parameters of the work tool, and to choose these parameters so as to 
ensure implementation of agro-technical requirements [8-14]. In addition, in some cases, this analysis and the analysis of velocities and acceleration of various points of rotary work tool allow one to choose design and operating parameters of the rotary work tools, providing a more effective impact on the soil while reducing the specific energy consumption for the tillage [15-18].

The purpose of this paper is to conduct a kinematic analysis of the tillage disk to obtain the priori conditions that allow rational choosing of its design parameters and operation modes.

\section{MATERIALS AND METHODS}

From the analogy with the dihedral wedge, G. E. Listopad and F.M. Kanarev proposed to evaluate the soil ripping capability with elementary area of the work tool moulded surface with the angle value of $\theta$ between the normal vector to the area and the vector of absolute velocity of any point of the considered area [19]. They substantiated their proposal by the fact that reducing this angle increases the stress concentration in the soil, and therefore increases the likelihood of its destruction. According to their data, the angle in the most common designs of plows varies in the range of $45^{\circ} \pm 9^{\circ}$ and designs of rotary tiller; the most widespread in practice, the range of variation of this angle is $90^{\circ} \pm 9^{\circ}$.

Let us define the change range of angle $\theta$ for an inclined tillage disk, moving at an angle of attack.

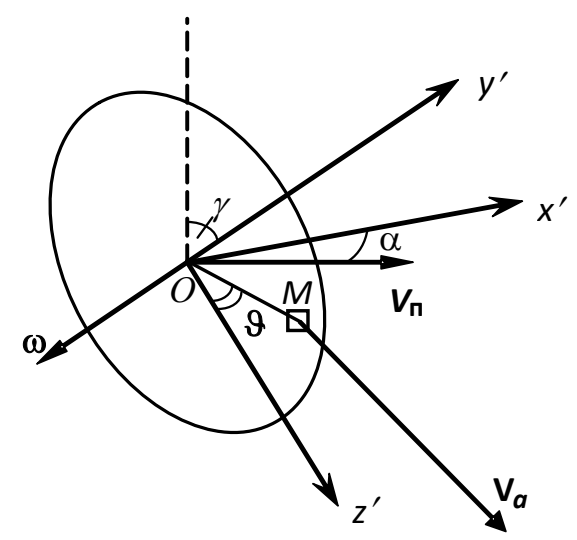

Fig. 1. Diagram to determine the absolute velocity of the disc blade point

The disc motion can be considered as complex: made up of constant speed $\mathbf{V}_{\text {п }}$ forward movement of the till-plow and rotary motion around the axis of the disk, with a certain constant angular velocity $\omega$.

Let us connect the disc center with the disc system of the moving axes $O x y^{\prime} z^{\prime}$, moving steadily at a speed $\mathbf{V}_{\text {п }}$, the axis $O x^{\prime}$ which will guide parallel to the straight line of intersection of the disc plane with the horizontal surface, the axis $O z^{\prime}$ in the disc plane perpendicular to the axis $O x^{\prime}$ down, and the axis $O y^{\prime}$ perpendicular to the disc plane in the direction which corresponds to right coordinate system (Fig. 1).
Elementary disc area is determined by its some point $M$, which position can be determined by its polar radius $\rho$ and angle $\vartheta$ between the radius vector of this point and positive direction of the axis $\mathrm{Oz}^{\prime}$, considering the positive direction of the reference angle - counterclockwise direction from the positive direction of axis $O y^{\prime}: M(\rho \sin \vartheta ; 0 ; \rho \cos \vartheta)$.

In case of direct rotation, the angular velocity vector of disk $\omega$ is directed along the axis of the disk in the direction of the soil that is opposite to the axis $O y^{\prime}$, therefore, in the present coordinate system it has coordinates $\omega=\{0 ;-\omega ; 0\}$.

Relative velocity $\mathbf{V}_{\mathrm{o}}$ of point $M$ is defined as the speed of the corresponding point of the disk, performing rotational movement around the axis $O y^{\prime}$ only. Since coordinates of the radius-vector of point $M$ are equal to its coordinates, then:

$$
\mathbf{V}_{\mathrm{o}}=\omega \times \boldsymbol{r}=\left|\begin{array}{ccc}
\boldsymbol{i} & \boldsymbol{j} & \boldsymbol{k} \\
0 & -\omega & 0 \\
\rho \sin \vartheta & 0 & \rho \cos \vartheta
\end{array}\right|
$$

Therefore:

$$
\mathbf{V}_{\mathrm{o}}=\omega \rho \cdot\{-\cos \vartheta ; 0 ; \sin \vartheta\} .
$$

Disk forward velocity vector in the chosen system of coordinates has the following coordinates:

$$
\mathbf{V}_{\Pi}=V_{\Pi} \cdot\{\cos \alpha ; \sin \alpha \sin \gamma ; \sin \alpha \cos \gamma\} \text {. }
$$

The vector of the absolute velocity $\mathbf{V}_{\mathrm{a}}$ of the point $M$ is determined as the geometric sum of the vectors $\mathbf{V}_{\mathrm{o}}$ and $\mathbf{V}_{\text {п }}$ and, because of (1) and (2):

$$
\mathbf{V}_{\mathrm{a}}=\mathrm{V}_{\Pi} \cdot\{\cos \alpha-\lambda \zeta \cos \vartheta ; \sin \alpha \sin \gamma ; \sin \alpha \cos \gamma+\lambda \zeta \sin \vartheta\},
$$

where $\lambda=\frac{\omega r}{\mathrm{~V}_{\Pi}}$ - the kinematic parameter that determines the operation mode of tillage disc $\zeta=\rho / r$. Since $r-h / \sin \gamma \leq \rho \leq r$, where $h$ is the maximum depth of tillage, then $1-\xi / \sin \gamma \leq \zeta \leq 1$, where $\xi=\frac{h}{r}$ is the relative depth of the disk.

The module of the absolute velocity vector of the point $M$ according to (3) is equal to:

$$
\mathrm{V}_{\mathrm{a}}=\sqrt{1+\lambda^{2} \zeta^{2}-2 \lambda \zeta(\sin \alpha \cos \gamma \sin \vartheta-\cos \alpha \cos \vartheta)} .
$$

Since the unit vector $\boldsymbol{j}$ of the axis $O y^{\prime}$ is perpendicular to the disk, then $\cos \theta=\frac{\mathbf{V}_{\mathrm{a}} \cdot \boldsymbol{j}}{\mathrm{V}_{\mathrm{a}}}$ :

$$
\cos \theta=\frac{\sin \alpha \sin \gamma}{\sqrt{1+u^{2}+2 u(\sin \alpha \cos \gamma \sin \vartheta-\cos \alpha \cos \vartheta)}},
$$

where $u=\lambda \varsigma$.

When tillage to a depth $(\max ) h$, angle $\vartheta$ varies in the range of $-\vartheta_{0} \leq \vartheta \leq \vartheta_{0}$, where $\vartheta_{0}=\arccos \left(1-\frac{\xi}{\sin \gamma}\right)$. 
Discs of diskers manufactured are usually vertical $(\gamma=$ $90^{\circ}$ ) and expression (5) simplifies:

$$
\cos \theta=\frac{\sin \alpha}{\sqrt{1+u^{2}-2 u \cos \alpha \cos \vartheta}},
$$

where $u=\lambda \varsigma$.

Angle $\alpha$ in such till-plows varies in the range of $\alpha=15^{\circ} \div 35^{\circ}[3]$.

Since the angle $\alpha$ is set and:

$$
\begin{aligned}
& \frac{\partial \cos \theta}{\partial \vartheta}=-\frac{u \sin \alpha \cos \alpha \sin \vartheta}{\sqrt{\left(1+u^{2}-2 u \cos \alpha \cos \vartheta\right)^{3}}}, \\
& \frac{\partial \cos \theta}{\partial u}=-\frac{\sin \alpha(u-\cos \alpha \cos \vartheta)}{\sqrt{\left(1+u^{2}-2 u \cos \alpha \cos \vartheta\right)^{3}}},
\end{aligned}
$$

the extreme value of the angle $\theta$ for vertical disk takes when:

$$
\left\{\begin{array}{c}
\sin \vartheta=0 \\
u=\cos \alpha \cos \vartheta
\end{array}\right.
$$

Hence $\vartheta=0, u=\cos \alpha, \theta_{\min }=0$.

Fig. 2. shows an angle $\theta$ - argument $u$-angle $\vartheta$ diagram when $\alpha=30^{\circ}$ for the vertical disc.

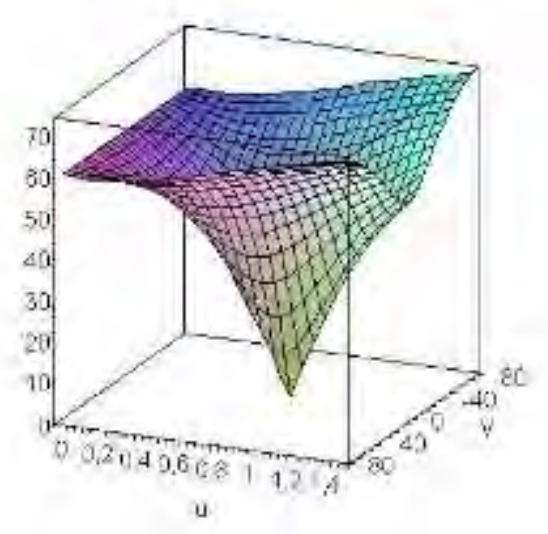

Fig. 2. Dependence of the angle $\theta$ on the argument $u$ and the angle $\vartheta$ on the angle $\gamma=90^{\circ}$

Thus for a fully slowed disc, when $\lambda=0$, angle $\theta=60^{\circ}$ for any its elementary area. With a fixed $\vartheta$ with the increase of the argument $u$, the angle $\theta$ values decrease to the value $u=$ $\cos \alpha \cos \vartheta$, and then start to increase. The minimum value of the angle $\vartheta$ is achieved when $\vartheta=0$. The absolute minimum of the angle $\theta$ equal to zero is achieved when the values $\vartheta=0$ and $u=\frac{\sqrt{3}}{2}$. Hence $\theta=0$ at $\lambda \varsigma \approx 0.866$. And when $\lambda>$ $0.866 / \varsigma$ angle $\theta$ increases with the increase of $\lambda$.

As per agro-technical requirements the primary tillage depth is $h=0.04 \div 0.06 \mathrm{~m}$ [3]. According to
V. F. Strelbitskiy diskers with a disc diameter of $D=0.45 \mathrm{~m}$ are the most widespread [3]. These values correspond to the relative depths $\xi=0.178 \div 0.267$. The calculations show that the maximum value of the angle $\theta$ in this case does not exceed $45^{\circ} \div 60^{\circ}$, which is significantly less than the value of this angle for the rotary tillers, and close to its value for plows. As per agricultural requirements, the discs of the disker should crumble the soil well enough [20]. Hence, the vertical discs satisfy this requirement.

The analysis shows that with the decrease of the disc tilt angle $\gamma$ the maximum value of the angle $\theta$ increases and for $\gamma=60^{\circ}$ at the same relative landing it is $60^{\circ} \div 68^{\circ}$. That is, with decreasing disc tilt angle, their digging capacity decreases.

The ability of tillage disks to efficiently perform technological functions and the required energy consumption are largely determined by the basic angles of cut sections of their blades. Let us define these angles and analyze their dependence on the geometric and operating parameters for a rational choice of values for these parameters.

For a model of compressible soil layer, the angle of cut with triangular wedge $\chi$ is determined by V.V. Blednikh [21] as the angle between the direction of movement of the soil wedge and the direction opposite to the direction of movement of the wedge, i.e. this angle completes the angle between the normal to the plane of the wedge and the vector of its forward speed to the angle is equal to $\pi / 2$ (see Fig. 3).

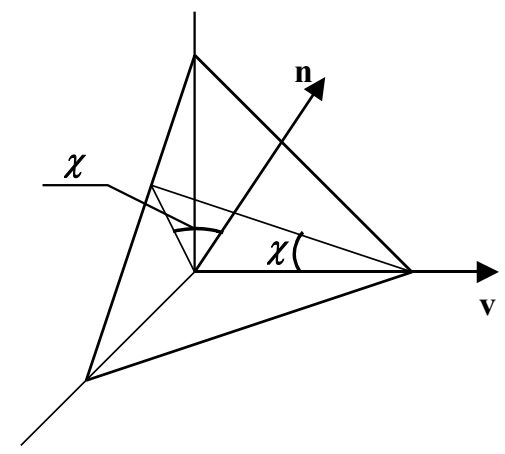

Fig. 3. Diagram to determine the cutting angle $\chi$ with a triangular wedge

Individual elementary sections of the flat disk blade can be considered as elementary triangular wedges with the common normal, which coincides with the normal to the disk, but moving at different absolute velocities, and therefore, having different cutting angles. As for blade, points $\rho=r$ and the cutting angle $\chi=\pi / 2-\theta$ (see Fig. 3), the sine of the cutting angle $\chi$ of elementary section of the blade, defined by the angle $\vartheta$, is determined from (5) when $\zeta=1$ :

$$
\sin \chi=\frac{\sin \alpha \sin \gamma}{\sqrt{1+\lambda^{2}+2 \lambda(\sin \alpha \cos \gamma \sin \vartheta-\cos \alpha \cos \vartheta)}},
$$

For vertical discs from (6), there is a simpler expression: 


$$
\sin \chi=\frac{\sin \alpha}{\sqrt{1+\lambda^{2}-2 \lambda \cos \alpha \cos \vartheta}} .
$$

The angle $\alpha$ value is determined by the agro-technical requirements. Having differentiated the last equality in private on variables $\vartheta$ and $\lambda$, we get:

$$
\begin{aligned}
& \frac{\partial \sin \chi}{\partial \vartheta}=-\frac{\lambda \sin \alpha \cos \alpha \sin \vartheta}{\sqrt{\left(1+\lambda^{2}-2 \lambda \cos \alpha \cos \vartheta\right)^{3}}}, \\
& \frac{\partial \sin \chi}{\partial \lambda}=-\frac{\sin \alpha(\lambda-\cos \alpha \cos \vartheta)}{\sqrt{\left(1+\lambda^{2}-2 \lambda \cos \alpha \cos \vartheta\right)^{3}}} .
\end{aligned}
$$

Therefore, the extreme value of the cutting angle $\chi$ takes when:

$$
\left\{\begin{array}{c}
\sin \vartheta=0 \\
\lambda=\cos \alpha \cos \vartheta
\end{array}\right.
$$

Hence $\vartheta=0, \lambda=\cos \alpha, \chi_{\max }=90^{\circ}$.

Fig. 4 shows a chart of dependence of angle $\chi$ on kinematic parameter $\lambda$ and angle $\vartheta$ when $\alpha=30^{\circ}$ for vertical disk.

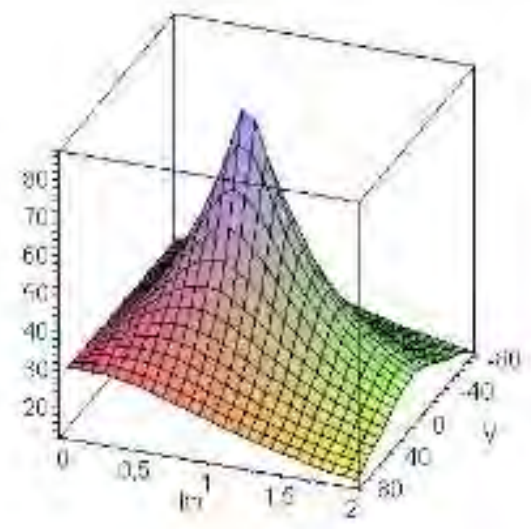

Fig. 4. The dependence of the angle $\chi$ on the parameter $\lambda$ and angle $\vartheta$ for angle $\gamma=90^{\circ}$

Based on this graph, when $\lambda>1$ the cutting angle of any elementary area of the blade decreases, prompting suggestions that the forced rotation disks provides lower specific energy consumption compared to passive disks.

Fig. 5 shows a chart of dependence of angle $\chi$ on kinematic parameter $\lambda$ and angle $\vartheta$ when $\alpha=30^{\circ}$ for the disk which is inclined to the surface of the field at the angle $\gamma=60^{\circ}$.

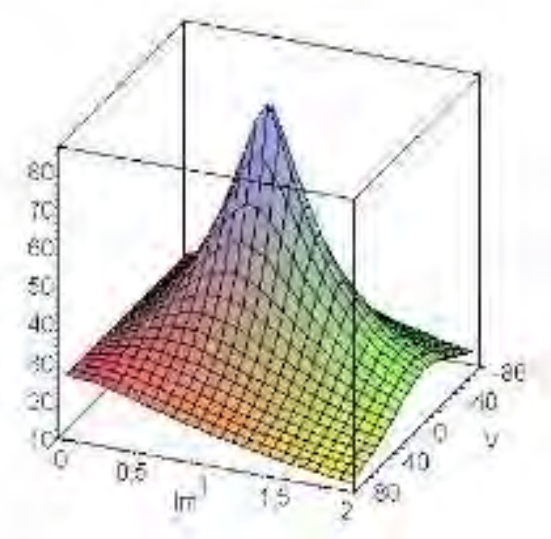

Fig. 5. The dependence of the angle $\chi$ from the parameter $\lambda$ and angle $\vartheta$ for angle $\gamma=60^{\circ}$

A comparison of the graphs in these figures shows that inclination of the disk shifts the maxima of the blade line element cutting angles in the direction of negative values $\vartheta$. A more detailed analysis shows that the cutting angles of the inclined disk for the most part of the blade is much lower $(20 \div 40 \%)$ of the respective cutting angles of a vertical disk. This result is consistent with the conclusion that the inclined disks are less energy-intensive according to the results of experimental studies by P. G. Kulebakin and A. I. Arzhanykh [4].

Let us find the lowest $\chi_{1}$ and maximum $\chi_{2}$ values of the cutting angle of the elementary sections of the blade.

As

$$
\frac{\partial(\sin \chi)}{\partial \vartheta}=-\frac{\lambda \sin \alpha \sin \gamma(\sin \alpha \cos \gamma \cos \vartheta+\cos \alpha \sin \vartheta)}{\sqrt{\left(1+\lambda^{2}+2 \lambda(\sin \alpha \cos \gamma \sin \vartheta-\cos \alpha \cos \vartheta)\right)^{3}}},
$$

the critical point is found from the equation:

$$
\sin \alpha \cos \gamma \cos \vartheta+\cos \alpha \sin \vartheta=0,
$$

or $\sin (\vartheta+\varepsilon)=0$, that is:

$$
\vartheta=-\varepsilon=-\operatorname{arctg}(\operatorname{tg} \alpha \cos \gamma) \text {. }
$$

The study of the derivative signs shows that this critical point is a maximum point, and given the fact that:

$$
\cos \varepsilon=\frac{\cos \alpha}{\sqrt{1-\sin ^{2} \alpha \sin ^{2} \gamma}}, \sin \varepsilon=\frac{\sin \alpha \cos \gamma}{\sqrt{1-\sin ^{2} \alpha \sin ^{2} \gamma}}
$$

from (6) and (7) we find the sine of the maximum cutting angle of the elementary section of the blade:

$$
\sin \chi_{2}=\frac{\sin \alpha \sin \gamma}{\sqrt{1+\lambda^{2}-2 \lambda \sqrt{1-\sin ^{2} \alpha \sin ^{2} \gamma}}},
$$

on the condition:

$$
\operatorname{arctg}(\operatorname{tg} \alpha \cos \gamma) \leq \arccos \left(1-\frac{\xi}{\sin \gamma}\right) .
$$


In this case, for vertical discs $(\sin \gamma=1, \cos \gamma=0)$ the sine of the maximum cutting angle of the elementary section of the blade is achieved when $\vartheta=0$, i.e., for the elementary section of the blade adjacent to the most recessed point of the disc, and is:

$$
\sin \chi_{2}=\frac{\sin \alpha}{\sqrt{1+\lambda^{2}-2 \lambda \cos \alpha}},
$$

on the condition:

$$
0 \leq \arccos (1-\xi),
$$

which occurs in case of any relative depth.

Given that $\lambda \geq 0$, and since:

$$
\frac{\partial \sin \chi_{2}}{\partial \lambda}=-\frac{\sin ^{2} \alpha \sin ^{2} \gamma\left(\lambda-\sqrt{1-\sin ^{2} \alpha \sin ^{2} \gamma}\right)}{\left(\sqrt{\left(1+\lambda^{2}-2 \lambda \sqrt{1-\sin ^{2} \alpha \sin ^{2} \gamma}\right)^{3}}\right.}=0
$$

when $\lambda=\sqrt{1-\sin ^{2} \alpha \sin ^{2} \gamma}$, at given lead angle $\alpha$ and the angle of disc inclination $\gamma$, the most cutting angle $\chi_{2}$ this value $\lambda$ takes the value of $90^{\circ}$.

The smallest cutting angle corresponds to the value of $\vartheta=\vartheta_{0}$, i.e. the elementary section of the blade is adjacent to the front point located on the daylight surface of the disk. Based on (6):

$$
\left\{\begin{array}{c}
\sin \chi_{1}=\frac{\sin \alpha \sin \gamma}{\sqrt{1+\lambda^{2}+2 \lambda\left(\sin \alpha \cos \gamma \sin \vartheta_{0}-\cos \alpha \cos \vartheta_{0}\right)}}, \\
\vartheta_{0}=\arccos \left(1-\frac{\xi}{\sin \gamma}\right) .
\end{array}\right.
$$

For the case of disks, located in the vertical planes, from (10) when $\sin \gamma=1$, we get:

$$
\sin \chi_{1}=\frac{\sin \alpha}{\sqrt{1+\lambda^{2}-2 \lambda \cos \alpha+2 \lambda \cos \alpha \xi}} .
$$

From experimental works on dihedral wedge we known range of optimum angles of soil cutting [22]:

$$
\chi_{\min } \leq \chi \leq \chi_{\max } .
$$

Therefore, tillage disc will operate at the lowest specific energy consumption when the following two conditions:

$$
\left\{\begin{array}{l}
\sin \chi_{\min } \leq \sin \chi_{1} \\
\sin \chi_{2} \leq \sin \chi_{\max }
\end{array}\right.
$$

where the sines of the angles $\chi_{1}$ and $\chi_{2}$ are determined for inclined disks by equations (8) and (10), and for vertical equations (9) and (11).

These terms can be used for a rational selection of geometrical parameters and operating conditions of the disc: they act as functional limitations that allow one to narrow the choice of parameters and to reduce the required number of laboratory and field experiments, requiring a long time and significant material costs for the implementation.

Inequations (12) can be interpreted as follows, the allowable range of parameters variation is determined for inclined disks $\left(\gamma<90^{\circ}\right)$ with surfaces of functions (8) and (10) level, and for vertical disks $\left(\gamma=90^{\circ}\right)$ with surfaces of functions (9) and (11) level.

Let us consider in detail a simpler case of vertical discs. In this case, it is easy to see that the level line for $\sin \chi_{2}=\sin \chi_{\max }$ consists of two branches, which are set by the following equations:

$$
\lambda_{1,2}=\frac{\sin \left(\chi_{\max } \pm \alpha\right)}{\sin \chi_{\max }}
$$

And two branches of level line for the $\sin \chi_{1}=\sin \chi_{\min }$ have the form

$$
\lambda_{3,4}=(1-\xi) \cos \alpha \pm \sqrt{(1-\xi)^{2} \cos ^{2} \alpha+\frac{\sin ^{2} \alpha}{\sin ^{2} \chi_{\text {min }}}-1} .
$$

Conditions (12) with (13) and (14) in this case can be written as follows:

$$
\left\{\begin{array}{c}
0 \leq \lambda \leq \frac{\sin \left(\chi_{\max }-\alpha\right)}{\sin \left(\chi_{\max }\right)} ; \\
\lambda \geq \frac{\sin \left(\chi_{\max }+\alpha\right)}{\sin \left(\chi_{\max }\right)} ; \\
(1-\xi) \cos \alpha-\sqrt{(1-\xi)^{2} \cos ^{2} \alpha+\frac{\sin ^{2} \alpha}{\sin ^{2} \chi_{\min }} \leq \lambda} \\
\lambda \leq(1-\xi) \cos \alpha+\sqrt{(1-\xi)^{2} \cos ^{2} \alpha+\frac{\sin ^{2} \alpha}{\sin ^{2} \chi_{\min }}}
\end{array}\right.
$$

Let us assume for certainty that $\xi=0,4, \chi_{\min }=15^{\circ}, \chi_{\max }=30^{\circ}$.

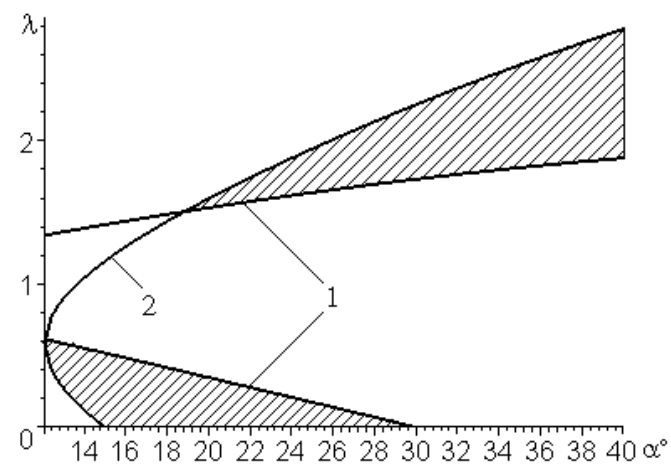

Fig. 6. Parameters region corresponding to the optimal blade line element cutting angle of disc

Fig. 4 consists of: 1 - two branches of level line $\sin \chi_{1}=\sin \chi_{\min }$ and $2-$ two branches of the line level $\sin \chi_{2}=\sin \chi_{\max }$. Biconnected domain of permissible values defined by inequalities (15) is shown by hatching. This figure shows that to get an optimal range of all blade line element 
cutting angles, interacting with the soil, one need either brake disks that are hard enough $(\lambda \leq 0,6)$ or force them to rotate $(\lambda \geq 1,4)$. In addition, the lead angles of the disks in the braking operation can be up to 1.5-2 times lower than lead angles of forced rotation modes.

\section{CONCLUSIONS}

This study allows drawing the following conclusions:

1. Vertical discs crumble the soil better than inclined ones. For small depth of the angle, characterizing ripping capability, they are closer to plows and go far beyond rotary tillers.

2. The methodology for the calculation of blade line element cutting angle of disc is set.

3. For an optimal range of all blade line element cutting angles, interacting with the soil, one need either brake disks that are hard enough or force them to rotate.

\section{References}

[1] V.F. Strelbitsky, "Power characteristics of flat and spherical disks of tillplows", Tractors and agricultural machinery, No. 8, pp. 28-31, 1970.

[2] N. V. Krasnoshchekov, "Disk tools to work at higher speeds", Mechanization and electrification of socialist agriculture, No. 4, pp. 2223, 1962.

[3] V.F. Strelbitsky, Disc tillage machines M: Mechanical engineering, 1978, 135 p., Ill.

[4] P. G. Kulebakin, A. I. Arzhanykh, "Study of the work of the till-plow with angled flat discs at higher speeds", Tractors and agricultural machinery, No. 4, pp. 29-31, 1965

[5] V. P. Goryachkin, Collected edition, in 3 vol., 2 ed, vol. 1, M: Kolos, 1968 , p. 720

[6] F.M. Kanarev, Rotary tillage machines and tools, M.: Mechanical engineering, 1983, 142 p., Ill.

[7] P. P. Khamitov, R. K. Abdrakhmanov, G. G. Bulgariev, M. N. Kalimullin, "Kinematics of a toothed rotating work tool Bulletin of Kazan state agrarian UNIVERSITY", № 3 (41), pp. 67-70, 2016.

[8] H. Guo, T. H. Burkhardt, R. H. Wilkinson, M. Hoki T. Tanoue, "Disk trajectory simulation of a powered disk tiller", Agricultural Mechanisation: Proceedigs of the Eleventh International Congress on Agricultural Engineering, pp. 1547-1553, Dublin, 4-8 September 1989.
[9] M. Amantayev, G. Gaifullin, R. Kravchenko, V. Kushnir, and S. Nurushev, "Investigation of the furrow formation by the disc tillage tools", Bulgarian Journal of Agricultural Science, vol. 24 (no 4) pp. 704 $709,2018$.

[10] G. Z. Gaifullin, L. A. Muntaeva, T. A. Murzabekov, M. A. Amantayev, "Definition of the plow sole formed by the rotary work tool", International agro- engineering, issue 4, pp. 35-40, 2012.

[11] V.V. Vasilenko, S. V. Vasilenko, V.V. Sheredekin, K. I. Kotlyakov, "Kinematics of the teeth of circular harrow", Bulletin of the Voronezh state agrarian UNIVERSITY, № 1 (52), pp. 129-134, 2017.

[12] V. I. Kazakov, I. V. Kazakov, "Kinematics and parameters of process of rolling of the rotary work tools of the cultivator for farm", Forestry journal, No. 3, pp. 156-160, 2016

[13] A. V. Ponomarev, "Kinematics of the wheel spider", the Scientific journal of the Kuban state agrarian University, № 97 (03), pp. 1-11, 2014

[14] M. S. Puzyrev, B. G. Kotlyar, V. A. Strizhov, "Research of quality and energy parameters of the double-disc rotary tillage work tool", Achievements of science and technology, No. 2, pp. 36-38, 2006

[15] M. J. O Dogherty, R. J. Godwin, A. P. Dedousis, J. L. Brighton, N. D. Tillett, "A Mathematical Model of the Kinematics of a Rotating Disc for Inter - and Intra-row Hoeing", Biosystems Engineering Vol. 96 (2), pp. $169-179,2007$.

[16] Zhao Xu , Chen Dong, "Optimal design of cutting-disc of no-tillage planter based on kinematics analysis and FEA", MATEC Web of Conferences40, 02015, 2016

[17] R. H. Gainutdinov, R. Kh. Aliakberov, G.V. Bikmullin, "Kinematics of the elliptical disc rotary tool for surface tillage", Machinery and equipment for the village, No. 8, pp. 10-14, 2016

[18] D. Yu. Kartashov, S. A. Vasilyev, E. P. Alekseev, A. A. Vasiliev, V. V. Alekseev, "Analysis of the teeth motion path when creating experimental tillage work tool", Bulliten of ChSPU n.a. I. Ya. Yakovlev, No. 4 (80), Part 2, pp. 91-94, 2013.

[19] G. E. Listopad, F.M. Kanarev, "On the deformation of soil by the work tools of tillage tools", lect. The All-Union Academy of Agricultura Sciences, No. 10, pp. 42-44, 1973.

[20] E. I. Trubilin, K. A. Sokht, V. I. Konovalov, S. V. Belousov, "Disc harrows and till-plows in the system of primary and secondary tillage. Problems and solutionы", Scientific journal of the Kuban state agrarian University, No. 88 (04), pp. 1-10, 2013.

[21] V.V. Blednikh, "Basic laws of force interaction of a triangular wedge with soil", Achievements of science and technology, No. 8, pp. 33-36, 2008.

[22] I. Z. Bagirov, "Impact of the wedge parameters on a cutting force", Academic conference works of TSNIIMASH of nonchernozem belt of the USSR in 1962, Minsk, 1962. 